\title{
SEM, EDS and CL Investigations of ZnMnO and ZnCoO Layers Grown at Low Temperature by Atomic Layer Deposition
}

\author{
M. Godlewski ${ }^{*, *}$, B. Witkowski ${ }^{*}$, B.J. Kowalski ${ }^{*}$, M. Łukasiewicz ${ }^{*}$, R. Jakieła ${ }^{*}$, E. Guziewicz ${ }^{*}$ \\ *Inst. of Physics, Polish Academy of Sciences, Al. Lotników 32/46, 02-668 Warsaw, Poland \\ ** Dept. Mathematics and Natural Sciences College of Science, Cardinal S. Wyszyński University, \\ Warsaw, Poland
}

Bulk samples, thin films and quantum dots of II-TM-VI and III-TM-V semiconductor alloys (where TM stands for a transition metal) are intensively studied for their possible spintronics applications. Room temperature ferromagnetism (RT FM) should be achieved in these diluted magnetic semiconductors (DMS) to allow for application of these materials in practical devices. This is an ultimate goal of the investigations.

RT FM was theoretically predicted for GaMnN and ZnMnO [1] (and then for other ZnTMO samples), which thus became the most studied spintronics materials. Soon after several groups reported $\mathrm{RT} F \mathrm{FM}$ of $\mathrm{ZnMnO}$ and $\mathrm{ZnCoO}$ (see e.g. [2]), but this is believed now to be due to inclusions of foreign phases, which we also demonstrate in the present work, and metal accumulations rather than volume properties of these two ZnTMO materials (see e.g. [3,4] and references given there).

Origin of the observed magnetic ordering remains often not clear. In the present work we employed scanning electron microscopy (SEM), energy dispersive X-rays spectroscopy (EDS) and cathodoluminescence (CL) methods to investigate topography, uniformity (we also used secondary ions mass spectroscopy (SIMS)) and chemical composition of ZnTMO (TM stands here for Co and $\mathrm{Mn})$ layers grown at low temperature by Atomic Layer Deposition. Samples obtained at these conditions are uniform and show only paramagnetic response, as reported for $\mathrm{ZnMnO}$ in $[3,4]$.

We demonstrate that these ZnTMO samples show several surprising properties not observed in e.g. ZnTMS. For example, we observed that Mn doping efficiently quenches visible emission of $\mathrm{ZnO}$ [5]. We show that this process is still more efficient in $\mathrm{ZnCoO}$, indicating that both $\mathrm{Mn}$ and Co ions act as emission deactivators in $\mathrm{ZnO}$. Emission quenching by $\mathrm{Mn}$ and $\mathrm{Co}$ allows us to study their distribution in $\mathrm{ZnO}$ from maps of in-plane changes of the CL intensity. We utilize the fact that the CL intensity anti-correlates with the TM concentration.

Strong magneto-optical effects are commonly seen in such DMS samples as CdMnTe. In CdMnTe excitonic emission shifts towards lower energy (by few meV) and splits for photoluminescence measured at different polarizations and magnetic field of a few $\mathrm{T}$ [6]. This was observed by us in CdMnTe layers [6] with a similar Mn concentration to the one studied by us at present in $\mathrm{ZnMnO}$. The efect was also reported for p-type $\mathrm{ZnMnO}$ layers [7]. Thus, in our magneto-optical investigations of n-type $\mathrm{ZnMnO}$ samples we looked for similar effects. Surprisingly, no spectral shifts and well-resolved splitting were observed for magnetic field up to $6 \mathrm{~T}$. 
We show that in-depth and in-plane CL mapping helps also to explain some of these puzzling magneto-optical properties of the ZnTMO films, related by us to the observed non-uniformity in TM distribution.

Acknowledgment

This work was partly supported by the FunDMS ERC Advanced Grant Research and by the European Union within European Regional Development Fund, through grant Innovative Economy (POIG.01.01.02-00-008/08).

The F-120 ALE reactor of Microchemistry was bought using SEZAM grant of the Foundation for Polish Science.

References

[1] T. Dietl, H. Ohno, F. Matsukura, J. Cibert, D. Ferrand, Science 287, (2000) 1019.

[2] Ü. Özgür, Ya.I. Alivov, C. Liu, A. Teke, M. Reshchikov, S. Dogan, V. Avrutin, S.-J. Cho, and H. Morkoc, J. Appl. Phys. 98, (2005) 041301.

[3] A. Wójcik, K. Kopalko, M. Godlewski, E. Guziewicz, R. Jakieła, R. Minikayev, W. Paszkowicz, Appl. Phys. Lett. 89, (2006) 051907.

[4] A. Wójcik, M. Godlewski, E. Guziewicz, R. Jakieła, M. Kiecana, M. Sawicki, M. Guziewicz, M. Putkonen, L. Niinistö, Y. Dumont, and N. Keller, Appl. Phys. Lett. 90, (2007) 082502.

[5] M. Godlewski, A. Wójcik-Głodowska, E. Guziewicz, S. Yatsunenko, A. Zakrzewski, Y. Dumont, E. Chikoidze, M.R. Phillips, Optical Materials 31, (2009) 1768.

[6] M. Godlewski, V.Yu. Ivanov, A. Khachapuridze, S. Yatsunenko, Phys. Stat. Sol. (b) 229, (2002) 533

[7] E. Przeździecka, E. Kamińska, M. Kiecana, M. Sawicki, Ł. Kłopotowski, W. Pacuski, J. Kossut, Solid State Commun. 139, (2006) 541. 